\title{
Electron Microscopy Revealed Massive Lipid Droplets in Cardiomyocytes in a Patient with Cardiogenic Shock Following a Fulminant Type 1 Diabetes Mellitus
}

\author{
Ryosuke Sugawara, ${ }^{1}$ MD, Hiroki Sugiyama, ${ }^{1,2}$ MD, Kazufumi Nakamura, ${ }^{2}$ MD, Kiyotaka Tohgi, ${ }^{3}$ MD, \\ Takashi Hongo, ${ }^{4}$ MD, Midori Tsuchiya, ${ }^{4} \mathrm{MD}$, Noriya Momoki, ${ }^{1} \mathrm{MD}$, Soichiro Nose, ${ }^{5} \mathrm{MD}$, \\ Chikao Yutani, ${ }^{6}$ MD, Yoshihiko Ikeda, ${ }^{7}$ MD, Tetsuya Ikeda, ${ }^{1}$ MD and Hiroshi Ito, ${ }^{2}$ MD
}

\begin{abstract}
Summary
A 52-year-old man with consciousness disorder following a 2-day history of general fatigue, diarrhea, vomiting and excessive thirst was admitted to our hospital. Severe hyperglycemia $(1,739 \mathrm{mg} / \mathrm{dL})$ with a slightly elevated $\mathrm{HbA} 1 \mathrm{c}$ level $(6.9 \%)$, ketonuria and low C-peptide level $(0.07 \mathrm{ng} / \mathrm{mL})$ confirmed the diagnosis of fulminant type 1 diabetes mellitus (FT1DM). Following sudden unexplained cardiogenic shock shortly after the initiation of insulin therapy with no evidence of myocardial ischemia assessed by coronary angiography, the patient was supported with percutaneous venoarterial extracorporeal membrane oxygenation. Electron microscopic analysis of the myocardium revealed massive lipid droplets without the infiltration of inflammatory cells. His left ventricular function began to recover during the following days and returned to a normal level on day 14 . Currently, the impact of FT1DM on intramyocardial lipid deposition is poorly understood. However, this case suggests that even short-term exposure to high concentrations of glucose can be responsible for lipotoxicity followed by severe cardiac dysfunction.
\end{abstract}

Key words: Lipotoxicity, Cardiac dysfunction, Diabetic cardiomyopathy

(Int Heart J 2021; 62: 197-200)

$\mathrm{F}$ ulminant type 1 diabetes mellitus (FT1D) is a subtype of type 1 diabetes mellitus characterized by the abrupt onset of severe hyperglycemia leading to diabetic ketoacidosis without the presence of isletrelated autoantibodies. ${ }^{1)}$ Previous studies have demonstrated that in rare instances, FT1D is accompanied by transient cardiac dysfunction diagnosed as myocarditis. ${ }^{2-5)}$ Viruses with overlapping infectivity in both the islet and myocardium were hypothesized to contribute to this simultaneous injury. ${ }^{\circ}$ However, it is noteworthy that cardiac abnormalities did not precede the onset of FT1D and were substantially displayed shortly after admission in these cases. There has been no mechanistic explanation for this stereotyped order of clinical onsets from the viewpoint of an infectious cause.

It has been recognized that one of the major mechanisms of diabetes-induced cardiac dysfunction is a maladaptive metabolic response in cardiomyocytes. ${ }^{7-9)}$ Longterm exposure to high glucose concentrations is responsible for myocardial lipid overload independent of the concentration of blood lipids that leads to non-ischemic cardiomyopathy, a phenomenon known as lipotoxicity. ${ }^{9-12)}$ How- ever, the impact of FT1DM, which involves abrupt and short-term hyperglycemic crises, on intramyocardial lipid deposition with severe cardiac dysfunction remains to be fully elucidated.

\section{Case Report}

An otherwise healthy 52-year-old man was brought to our emergency department because of consciousness disorder. He had a 2-day history of general fatigue, diarrhea and vomiting and complained of excessive thirst. There was no family history of note. On admission, his heart rate was 68 beats/min with a blood pressure of 91/ $70 \mathrm{mmHg}$. Arterial blood gas analysis showed $\mathrm{pH} 7.123$, $\mathrm{pCO}_{2} 22.8 \mathrm{mmHg}, \mathrm{pO} 278.1 \mathrm{mmHg}, \mathrm{O}_{2}$ saturation $92.5 \%$, and bicarbonate $7.2 \mathrm{mmol} / \mathrm{L}$. Blood tests showed severe hyperglycemia (a blood glucose of $1,739 \mathrm{mg} / \mathrm{dL}$ ). However, the $\mathrm{HbAlc}$ levels remained slightly elevated $(6.9 \%)$, which indicated a short-term exposure to hyperglycemia. Autoantibodies against glutamic acid decarboxylase and islet antigen-2 were negative. Pancreatic amylase serum levels were slightly elevated $(85 \mathrm{U} / \mathrm{L})$, but abdominal

From the ${ }^{1}$ Department of Internal Medicine, Okayama Saiseikai General Hospital, Okayama, Japan, ${ }^{2}$ Department of Cardiovascular Medicine, Okayama University Graduate School of Medicine, Dentistry and Pharmaceutical Sciences, Okayama, Japan, ${ }^{3}$ Department of Cardiology, The Sakakibara Heart Institute of Okayama, Okayama, Japan, ${ }^{4}$ Department of Emergency Medicine, Okayama Saiseikai General Hospital, Okayama, Japan, ${ }^{5}$ Department of Pathology, Okayama Saiseikai General Hospital, Okayama, Japan, ${ }^{6}$ Department of Pathology, Amagasaki Chuo Hospital, Amagasaki, Japan and ${ }^{7}$ Department of Pathology, National Cerebral and Cardiovascular Center, Suita, Japan.

Address for correspondence: Hiroki Sugiyama, MD or Kazufumi Nakamura, MD, Department of Cardiovascular Medicine, Okayama University Graduate School of Medicine, Dentistry and Pharmaceutical Sciences, 2-5-1 Shikata-cho, Kita-ku, Okayama 700-8558, Japan. E-mail: ichibun@cc.okayama-u.ac.jp Received for publication July 27, 2020. Revised and accepted September 28, 2020.

doi: 10.1536/ihj.20-537

All rights reserved by the International Heart Journal Association. 
A

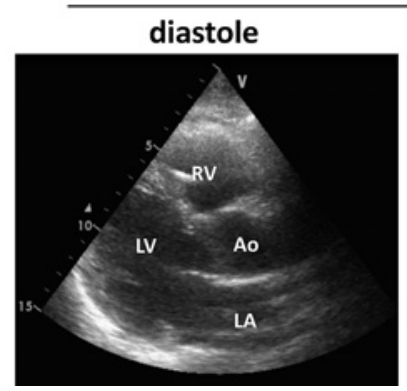

B

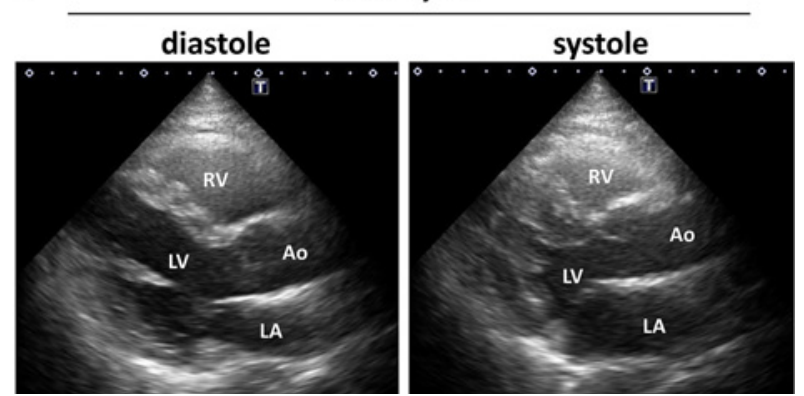

Figure 1. Transthoracic echocardiographic images on days 0 and 14. A: Images on day 0 show left ventricular contractile dysfunction. No findings suggestive of myocardial edema or epicardial effusion were detected. B: Images on day 14 show improvement in left ventricular contractile function.
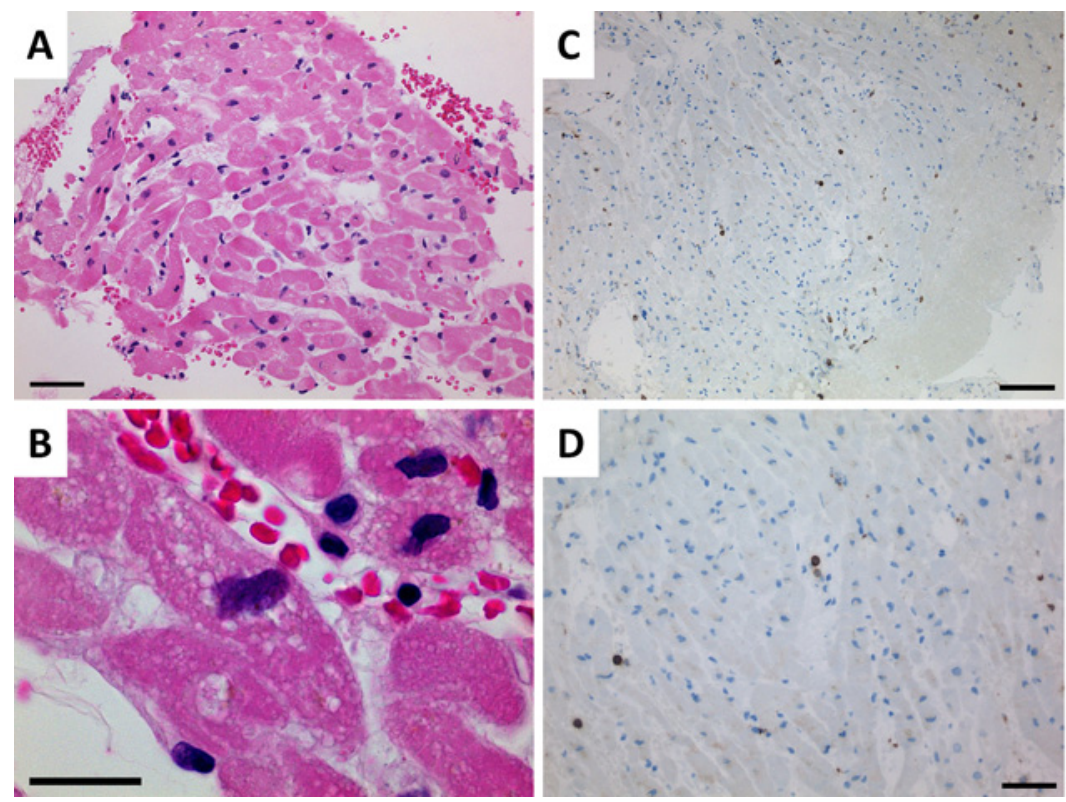
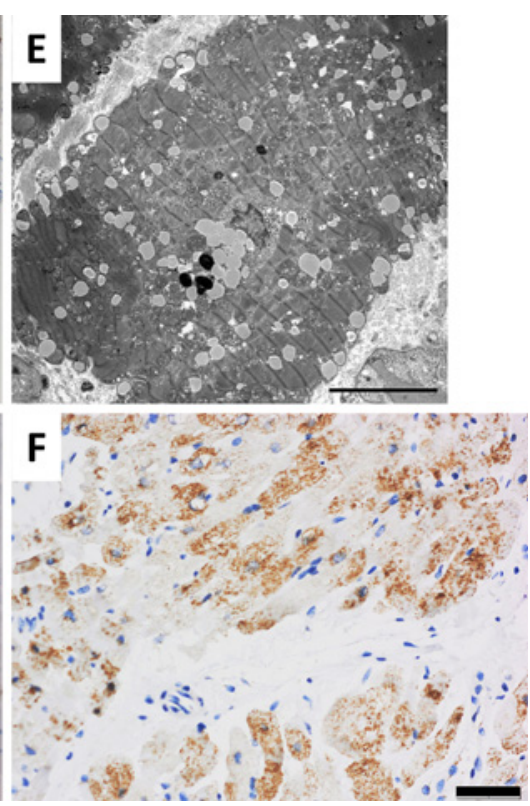

Figure 2. Cardiac biopsy findings. A, B: Hematoxylin and eosin staining. Small vacuoles are observed in the cytoplasm of cardiomyocytes. Bar $=50 \mu \mathrm{m}(\mathbf{A}), 20 \mu \mathrm{m}(\mathbf{B})$. C, D: Immunohistochemical staining with a CD45RB antibody. Bar $=100 \mu \mathrm{m}(\mathbf{C}), 50 \mu \mathrm{m}(\mathbf{D})$. E: Electron microscopic image. Massive lipid droplets are identified in the cytoplasm of cardiomyocytes. Bar $=5 \mu \mathrm{m}$. F: Immunohistochemical staining with an adipose triglyceride lipase (ATGL) antibody. ATGL expression is confirmed in the cytoplasm of cardiomyocytes. Bar $=50 \mu \mathrm{m}$.

computed tomography revealed no evidence of pancreatic diseases. Blood ketones were found to be elevated at 17.4 $\mathrm{mmol} / \mathrm{L}$, and urinalysis showed ketonuria. Serum peptide levels were also low $(0.07 \mathrm{ng} / \mathrm{mL})$. These findings confirmed the diagnosis of FT1D. An initial electrocardiogram (ECG) demonstrated no evidence of myocardial ischemia.

One hour after he was started on a continuous insulin infusion, ECG monitoring showed bradycardia accompanied by frequent pauses, and ventricular stimulation by external pacemakers was introduced. Transthoracic echocardiography (TTE) showed that left ventricle contraction remained within normal limits in this instance. The blood glucose level decreased to $639 \mathrm{mg} / \mathrm{dL}$ during the first 18 hours. However, it was becoming more difficult to maintain normal blood pressure despite fluid resuscitation and combined inotropic/vasopressor therapy. TTE at that time revealed severe cardiac dysfunction [left ventricular enddiastolic diameter (LVDd)/left ventricular end-systolic diameter (LVDs), 48/44 $\mathrm{mm}$ and left ventricular ejection fraction (LVEF), 16\%], which indicated cardiogenic shock (Figure 1A). Mechanical circulatory support with percutaneous venoarterial extracorporeal membrane oxygenation and intra-aortic balloon pumping were initiated. Although laboratory findings showed enhanced levels of creatine kinase (CK) (2,833 IU/L), CK-MB isoenzyme (83 IU/L) and troponin-I $(11,383 \mathrm{pg} / \mathrm{mL})$, coronary angiography did not show any evidence of coronary artery disease.

Hematoxylin and eosin staining (Figure 2A and B) and immunohistochemical staining with a CD45RB antibody (Figure 2C and D) of endomyocardial biopsy samples (a total of 4 specimens) revealed small vacuoles in the cytoplasm of cardiomyocytes and only a minority of inflammatory cell infiltration without edema or fibrosis in 
the myocardium. Electron microscopy analyses of the myocardium showed massive lipid droplets in the cytoplasm of cardiomyocytes (Figure 2E). Immunohistochemical studies confirmed the presence of adipose triglyceride lipase (ATGL) (Figure 2F). The peak levels of CK, CK$\mathrm{MB}$, troponin-I and B-type natriuretic peptide during the course of illness were 3,221 IU/L on day 3. $89 \mathrm{IU} / \mathrm{L}$ on day $1,24,020 \mathrm{pg} / \mathrm{mL}$ on day 4 , and $371.9 \mathrm{pg} / \mathrm{mL}$ on day 2 , respectively.

Left ventricular systolic function began to recover during the following days, and the patient was successfully weaned off mechanical circulatory support on day 9 . TTE revealed that left ventricular function returned to a normal level on day 14 (LVDd/LVDs, 43/25 $\mathrm{mm}$ and LVEF, 73\%; Figure 1B).

\section{Discussion}

The main significant finding of this case report is that massive lipid droplets in cardiomyocytes are present in the acute phase of FT1D complicated by the abrupt and serious but completely reversible cardiogenic shock. A general model for the development of non-ischemic diabetic cardiomyopathy includes the excessive reliance on fatty acids (FAs) for energy production leading to the accumulation of lipids in the heart (so-called lipotoxicity). ${ }^{13-15)}$ At present, it remains unknown whether cardiac steatosis leading to massive lipid droplets emerged as an acute phase response to FT1DM. Another point to note is that cardiac function deteriorated shortly after the initiation of insulin therapy and was not present on admission. Developments leading up to the fulminant heart failure occurred during the first several days after admission, showing that our case is consistent with previous reports of FT 1D complicated by myocarditis. ${ }^{2-5)}$ However, pathological findings in our case ruled out the possibility of inflammatory tissue injury. Based on these observations, metabolic abnormalities may be implicated as a leading cause of the myocardiopathy.

The abundant neutral lipid droplets in cardiomyocytes is a hallmark of triglyceride deposit cardiomyovasculopathy (TGCV), a chronic systemic disease that presents as severe cardiac dysfunction often requiring subsequent heart transplantation. ${ }^{16,17)} \mathrm{A}$ prominent feature of its pathophysiology is the disruption of long-chain fatty acid (LCFA) metabolism, which is caused by abnormalities in ATGL or other functional components of metabolic pathways. ${ }^{18,19)}$ A histopathological examination of this patient excluded the possibility of primary TGCV caused by a genetic deficiency in ATGL. Conversely, idiopathic TGCV, which shows abnormal LCFA metabolism without ATGL gene-deficiency, is thought to be a possible cause of the combination of severe cardiac dysfunction and abnormal accumulation of intramyocardial lipid droplets. ${ }^{20)}$ However, the specific clinical characteristics of TGCV, including slow-progressive and irreversible cardiac dysfunction and the development of diffuse coronary arteriosclerosis, differ from our case in many ways $\mathrm{s}^{21)}$.

Diabetes mellitus is associated with cardiac dysfunction, even in the absence of coronary atherosclerosis. The incorporation of excess free FAs into the myocardium promotes oxidative stress, insulin resistance and mitochondrial dysfunction through an increase in lipotoxic intermediates, such as ceramide and diacylglycerol. ${ }^{22}$ Previous studies have demonstrated that the upregulated gene expression of peroxisome proliferator-activated receptor (PPAR) alpha, which is responsible for myocardial fatty acid use, leads to enhanced lipotoxicity in diabetic cardiomyopathy. ${ }^{23)}$ However, for nondiabetic patients, there were inconsistent associations between PPAR alpha expression levels and cardiac dysfunction; therefore, it is unclear how the regulation of FA metabolism contributes to the pathogenesis of heart failure. ${ }^{24)}$

Current evidence does not provide an answer to the fundamental question of why cardiac dysfunction appeared at a rapid rate immediately after the start of treatment. In our case, a distinctive feature of the metabolic aspect is the drastic switching of energy substrates between FAs and glucose during the clinical course. Accordingly, it is assumed that the unsuccessful modulation of energy use is a potential mechanism of FT1DM-associated abrupt deterioration in myocardial function. FT1DM is characterized by rapid and severe depletion of insulin, indicating glucose starvation followed by significant inhibition of glucose metabolic pathways in cardiomyocytes via the Randle cycle. ${ }^{1,25)}$ A key step in the alteration of intracellular metabolic regulation triggered by insulin reintroduction is the rapid stimulation of glucose transporter 4 translocation and subsequent extensive glucose influx into cardiomyocytes within 30 minutes of insulin administration. ${ }^{26)}$ Under the condition of impaired glucose oxidation, a high rate of glucose uptake may overwhelm the rate of glucose oxidation and lead to the accumulation of glycolytic intermediates and advanced glycation end-productinduced reactive oxygen species involved in the initiation of apoptosis. ${ }^{27)}$ Furthermore, it is noteworthy that subsequent high concentrations of intracellular glucose also provoke the inhibition of FA oxidation in the heart through the repression of PPAR alpha and related genes, suggesting the complete deprivation of principal energy sources. ${ }^{28)}$ This hypothesis for metabolic adaptation is supported by a previous study demonstrating fatal cardiac injury and accumulated neutral lipids in cardiomyocytes in conditional PPAR alpha-knockout mice during fasting. ${ }^{29)}$

The main limitations of this case report include the lack of histological follow-up, not feasible for ethical reasons, and certification for improvement of cardiac lipid metabolism. Further studies are needed to elucidate whether the myocardial lipid accumulation is reduced in accordance with recovery of cardiac function. Previous experiments had suggested substantial influence of systemic or local inflammatory response on myocardial lipid accumulation. ${ }^{13)}$ Severe cardiac dysfunction itself is also positively associated with lipid droplets in the electron microscopy of the cardiomyocytes. A wide variety of diseased conditions would be taken into consideration in the pathogenesis of this case.

The elevation of serum enzymes including CK, CKMB and troponin-I seems inconsistent with a lack of histopathological features of myocyte necrosis. However, CK-MB is a very low percentage of total CK in our case, which indicate high probabilities of skeletal muscle dam- 
age evoked by diabetic ketoacidosis. Furthermore, the cellular pathophysiology of cardiac troponin release includes enhanced cell permeability followed by the extrusion from reversibly injured myocytes in the absence of cell death. ${ }^{30}$

\section{Conclusion}

In this report, we presented a case of FT1DM followed by the abrupt onset of severe transient cardiac dysfunction and massive lipid droplets in cardiomyocytes, a characteristic pathological feature common to TGCV, detected in a heart biopsy sample. Without follow-up histopathological examinations of the myocardium in the convalescent phase, we can only make inferences about whether lipid droplet formation is coincident with the onset of severe contractile dysfunction in our case. Moreover, the causal relationship between lipid deposition and cardiac dysfunction also remains uncertain. However, our observation provides the concept of metabolic maladaptation associated with insulin-mediated regulation of energy substrate use among patients with FT1DM.

\section{Acknowledgments}

The authors are grateful to Masayo Ohmori, Miyuki Misunaga and Kaoru Akazawa for their excellent technical assistance. We thank Melissa Crawford, PhD, from Edanz Group (https://en-author-services.edanzgroup.com/ac) for editing a draft of this manuscript.

\section{Disclosure}

Conflicts of interest: The authors declare that there is no conflict of interest.

\section{References}

1. Imagawa A, Hanafusa T, Miyagawa J, Matsuzawa Y. A novel subtype of type 1 diabetes mellitus characterized by a rapid onset and an absence of diabetes-related antibodies. Osaka IDDM Study Group. N Engl J Med 2000; 342: 301-7.

2. Hiramatsu S, Komori K, Mori E, Ogo A, Maruyama S, Kato S. A case of fulminant type 1 diabetes mellitus accompanied by myocarditis. Endocr J 2011; 58: 553-7.

3. Makino K, Nishimae I, Suzuki N, et al. Myocarditis with fulminant type 1 diabetes mellitus diagnosed by cardiovascular magnetic resonance imaging: a case report. BMC Res Notes 2013; 6: 347 .

4. Ohara N, Kaneko M, Kuwano H, et al. Fulminant type 1 diabetes mellitus and fulminant viral myocarditis. A case report and literature review. Int Heart J 2015; 56: 239-44.

5. Mokuno T, Sawai Y, Oda N, et al. A case of myocarditis associated with IDDM. Diabetes Care 1996; 19: 374-8.

6. Yoneda S, Imagawa A, Fukui K, et al. A Histological Study of Fulminant Type 1 Diabetes Mellitus Related to Human Cytomegalovirus Reactivation. J Clin Endocrinol Metab 2017; 102 2394-400.

7. Boudina S, Abel ED. Diabetic cardiomyopathy revisited. Circulation 2007; 115: 3213-23.

8. Taegtmeyer H, McNulty P, Young ME. Adaptation and maladaptation of the heart in diabetes: Part I: general concepts. Circula- tion 2002; 105: 1727-33.

9. Young ME, McNulty P, Taegtmeyer H. Adaptation and maladaptation of the heart in diabetes: Part II: potential mechanisms. Circulation 2002; 105: 1861-70.

10. McGavock JM, Lingvay I, Zib I, et al. Cardiac steatosis in diabetes mellitus: a 1H-magnetic resonance spectroscopy study. Circulation 2007; 116: 1170-5.

11. Ruberg FL. Myocardial lipid accumulation in the diabetic heart. Circulation 2007; 116: 1110-2.

12. Shimabukuro M. Cardiac adiposity and global cardiometabolic risk: new concept and clinical implication. Circ J 2009; 73: 2734.

13. Sharma S, Adrogue JV, Golfman L, et al. Intramyocardial lipid accumulation in the failing human heart resembles the lipotoxic rat heart. FASEB J 2004; 18: 1692-700.

14. Toth PP, Raghavan VA. Glucolipotoxicity and the heart. Heart Fail Clin 2012; 8: xvii-xviii.

15. Nakanishi T, Kato S. Impact of diabetes mellitus on myocardial lipid deposition: an autopsy study. Pathol Res Pract 2014; 210: 1018-25.

16. Hirano K, Ikeda Y, Zaima N, Sakata Y, Matsumiya G. Triglyceride deposit cardiomyovasculopathy. N Engl J Med 2008; 359: 2396-8.

17. Hirano K. A novel clinical entity: triglyceride deposit cardiomyovasculopathy. J Atheroscler Thromb 2009; 16: 702-5.

18. Haemmerle G, Lass A, Zimmermann R, et al. Defective lipolysis and altered energy metabolism in mice lacking adipose triglyceride lipase. Science 2006; 312: 734-7.

19. Zimmermann R, Strauss JG, Haemmerle G, et al. Fat mobilization in adipose tissue is promoted by adipose triglyceride lipase. Science 2004; 306: 1383-6.

20. Takagi A, Ikeda Y, Kobayashi K, et al. Newly developed selective immunoinactivation assay revealed reduction in adipose triglyceride lipase activity in peripheral leucocytes from patients with idiopathic triglyceride deposit cardiomyovasculopathy. Biochem Biophys Res Commun 2018; 495: 646-51.

21. Ikeda Y, Hirano K, Fukushima N, Sawa Y. A novel type of human spontaneous coronary atherosclerosis with triglyceride deposition. Eur Heart J 2014; 35: 875.

22. Jia G, Hill MA, Sowers JR. Diabetic Cardiomyopathy: An Update of Mechanisms Contributing to This Clinical Entity. Circ Res 2018; 122: 624-38.

23. Goldberg IJ, Trent CM, Schulze PC. Lipid metabolism and toxicity in the heart. Cell Metab 2012; 15: 805-12.

24. Barger PM, Brandt JM, Leone TC, Weinheimer CJ, Kelly DP. Deactivation of peroxisome proliferator-activated receptor-alpha during cardiac hypertrophic growth. J Clin Invest 2000; 105: 1723-30.

25. Randle PJ, Garland PB, Hales CN, Newsholme EA. The glucose fatty-acid cycle. Its role in insulin sensitivity and the metabolic disturbances of diabetes mellitus. Lancet 1963; 1: 785-9.

26. Czech MP, Corvera S. Signaling mechanisms that regulate glucose transport. J Biol Chem 1999; 274: 1865-8.

27. Rosen P, Du X, Tschope D. Role of oxygen derived radicals for vascular dysfunction in the diabetic heart: prevention by alphatocopherol? Mol Cell Biochem 1998; 188: 103-11.

28. Roduit R, Morin J, Masse F, et al. Glucose down-regulates the expression of the peroxisome proliferator-activated receptoralpha gene in the pancreatic beta -cell. J Biol Chem 2000; 275 : 35799-806.

29. Leone TC, Weinheimer CJ, Kelly DP. A critical role for the peroxisome proliferator-activated receptor alpha (PPARalpha) in the cellular fasting response: the PPARalpha-null mouse as a model of fatty acid oxidation disorders. Proc Natl Acad Sci U S A 1999; 96: 7473-8.

30. Mair J, Lindahl B, Hammarsten O, et al. How is cardiac troponin released from injured myocardium? Eur Heart J Acute Cardiovasc Care 2018; 7: 553-60. 